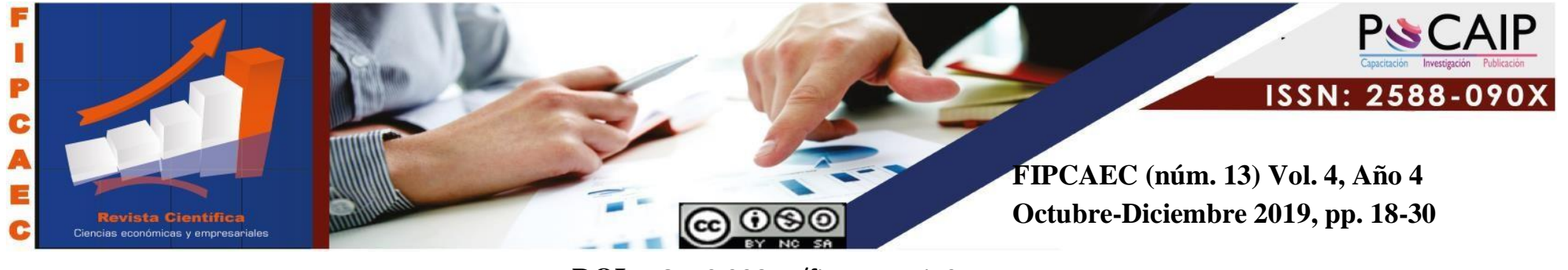

DOI: DOI 10.23857/fipcaec.v4i12.77

\title{
Programa de entrenamiento en liderazgo gerencial en organizaciones empresariales
}

\section{Management leadership training program in business organizations}

Programa de treinamento em liderança gerencial em organizações empresariais

\author{
Henry Darío Suárez-Vélez I \\ pocaipecuador@gmail.com \\ https://orcid.org/0000-0002-6817-2867
}

\author{
Marcos Boanerge Vera-Mendoza ${ }^{\mathrm{I}}$ \\ mveram88@hotmail.com \\ https://orcid.org/0000-0002-0048-651X
}

Correspondencia: pocaipecuador@gmail.com

* Recepción: 21/ 07/ 2019 *Aceptación: 12/09/ 2019 *Publicación: 15 /10/ 2019

1 Licenciado en Trabajo Social, Polo de Capacitación, Investigación y Publicación (POCAIP), Manta, Ecuador.

2 Master Universitario en Formulación y Tecnología del Producto, Ingeniero Industrial, Docente de la Universidad Laica Eloy Alfaro de Manabí, Manta, Ecuador. 


\title{
Resumen
}

La investigación tuvo por objetivo determinar la eficacia de un programa de entrenamiento en liderazgo gerencial en organizaciones empresariales. La prueba fue aplicada a la población de la empresa suministros Klamer, ubicados en la ciudad de Portoviejo de la provincia de Manabí Ecuador, conformada por 63 personas, las cuales se dividieron en dos grupos al azar, comtem0plados del siguiente modo: G1 conformado por 31 personas y G2 conformado por 32 personas. En pos prueba el nivel de significancia bilateral cambió a ,026 en G2, lo que indica diferencias significativas. La organización cuenta con pensadores estratégicos que puedan mirar más allá de los muros de la organización, previniendo la articulación de acciones que permitan anticipar las acciones negativas que puedan sobrevenir, convirtiéndolas en oportunidades para crecer integralmente en favorecer no solo la productividad organizacional, sino el propio crecimiento personal como ente motivador para articular cada día mejores acciones.

Palabras clave: Liderazgo; delegación de autoridad; toma de decisiones; resolución de problemas.

\begin{abstract}
The research aimed to determine the effectiveness of a management leadership training program in business organizations. The test was applied to the population of Klamer Supplies Company, located in the city of Portoviejo in the province of Manabí - Ecuador, consisting of 63 people, which were divided into two random groups, as follows: G1 formed by 31 people and G2 made up of 32 people. In post-test the level of bilateral significance changed to, 026 in G2, indicating significant differences. The organization has strategic thinkers who can look beyond the walls of the organization, preventing the articulation of actions that allow anticipating the negative actions that may occur, turning them into opportunities to grow integrally in favoring not only organizational productivity, but their own personal growth as a motivating entity to articulate better actions every day.
\end{abstract}

Keywords: Leadership; delegation of authority; decision making; Problem resolution.. 


\section{Resumo}

A pesquisa teve como objetivo determinar a eficácia de um programa de treinamento em liderança gerencial em organizações empresariais. O teste foi aplicado à população da empresa de suprimentos Klamer, localizada na cidade de Portoviejo, na província de Manabí - Equador, composta por 63 pessoas, divididas em dois grupos aleatórios, a seguir: G1 formado por 31 pessoas e o G2 composto por 32 pessoas. No pós-teste, o nível de significância bilateral mudou para 026 no G2, indicando diferenças significativas. A organização possui pensadores estratégicos que podem olhar além dos muros da organização, impedindo a articulação de ações que permitem antecipar as ações negativas que podem ocorrer, transformando-as em oportunidades de crescimento integral, favorecendo não apenas a produtividade organizacional, mas também a sua própria. Crescimento pessoal como entidade motivadora para articular ações melhores todos os dias.

Palavras-chave: Liderança; delegação de autoridade; tomada de decisão; resolução de problemas.

\section{Introducción}

El liderazgo es un fenómeno social, porque se desarrolla, precisamente en grupos sociales, por tanto su estudio y conceptualización tiene una larga trayectoria, por ende ha adoptado diversas perspectivas, según los cambios que se generan en el mundo. La importancia del liderazgo en organizaciones empresariales, radica en que es un medio que permite la disposición de las personas al cambio, un cambio que muchas veces genera incertidumbre en el grupo, pero que al mismo tiempo es una oportunidad para fomentar y desarrollar en los miembros de una comunidad, la capacidad de reflexión, ante nuevos elementos que se presentan en el contexto, así como también, permite abrir e incluso crear espacios para lograr la organización, en este caso de la empresa.

Es importante mencionar que el liderazgo está estrechamente interrelacionado con la motivación, al mismo tiempo es un factor determinante en las gestiones de cambio e innovación, por tanto repercute de forma considerable en los procesos y en los resultados. Esto apunta a considerar la importancia que ha tomado el liderazgo como elemento y proceso fundamental para el desarrollo 
de la sociedad, por cuanto coadyuva en los procesos creativos y en el comportamiento de las personas en ambientes de convivencia o de trabajo (Chiavenato, 2006, p. 56)

El liderazgo supone procesos de cambios y para ello necesita de seguidores, la gente tiende a seguir a quienes le ofrecen medios para la satisfacción de sus deseos y necesidades. Según Koontz y Weihrich (1999) "el liderazgo es el arte o proceso de influir en las personas para que se esfuercen voluntaria y entusiastamente en el cumplimiento de metas grupales". Según Chiavenato (2006), el liderazgo es la influencia interpersonal ejercida en una situación, orientada a la consecución de uno o diversos objetivos específicos mediante el proceso de comunicación humana. Los autores hacen referencia que el liderazgo es un fenómeno social, por tanto ocurre exclusivamente en grupos sociales, esto quiere decir que el contexto cultural y las situaciones en que se encuentra un determinado grupo son determinante para el liderazgo, por tanto, no depende solo de la cualidad personal que hace de un individuo un líder, sino, de las decisiones que se toman de acuerdo a la situación que se presenta, de ahí radica la importancia del liderazgo como un factor que minimiza la incertidumbre del grupo.

En atención a las definiciones expuestas anteriormente, se observa que ambos autores utilizan la palabra influencia, de acuerdo a Chiavenanato (2006), es una fuerza psicológica que permite que una persona actúe con la intención de modificar el comportamiento de otra, de esto se traduce en que el liderazgo se basa en las relaciones entre las personas, entre grupos que se unen se acuerdo a sus luchas por satisfacer sus necesidades, por tanto las personas en todo momento buscarán los medios y las relaciones adecuadas, para solucionar problemas o hacer frente a sus necesidades. Por tanto según Chiavenato (2006), el liderazgo, que depende de las necesidades que se presentan en un determinado momento, es una relación entre un individuo y un grupo. Al mismo tiempo que establece lo siguiente: "el proceso de liderazgo es una función del líder, de los seguidores y de las variables de la situación”.

En tal sentido, para las organizaciones empresariales es muy importante desarrollar un liderazgo basado en las fortalezas de las personas, en sus habilidades y capacidades, para que sea posible promover mayor eficacia y productividad en los procesos inherentes a la organización, Isea- 
Argüelles \& Aldana-Zavala (2017), plantea que el liderazgo es de suma importancia para motivar al cumplimiento de actividades que permitan cumplir con las metas propuestas, siendo que este liderazgo debe ser de corte transformacional con la finalidad de promover una gestión de calidad continua, aunado a lo descrito, Rojas de Uzcátegui, Molleja \& Morles (2016), plantean la necesidad de contar con el auto liderazgo, por cuanto:

Es importante acotar que la gerencia actual demanda de gerentes capaces de poseer un enfoque integrador en sus estilos de pensamiento, que con el autocontrol, la autoevaluación y el autodescubrimiento, puedan direccionar el rumbo de las organizaciones frente a los niveles de incertidumbre, oscuridades y ruidos del complejo mundo en que vivimos (p. 15).

Es importante indicar la necesidad de que las organizaciones empresariales cuenten con líderes con visión sistémica con la finalidad de promover acciones complejas en favor de vincular soluciones desde las adversidades que puedan presentarse, Aldana, Salón \& Guzmán (2019), complementan al indicar la necesidad de que las organizaciones cuenten con "un liderazgo integrador en procura de obtener la mayor efectividad posible en el cumplimiento de las metas propuestas" (p. 50), de ese modo, la actual investigación se plantea determinar la eficacia de un programa de entrenamiento en liderazgo gerencial en organizaciones empresariales, en el contexto de la empresa suministros Klamer, ubicada en la ciudad de Portoviejo de la provincia de Manabí - Ecuador, con la finalidad de promover aptitudes relacionadas a un gerente sistémico en capacidad de promover desde la complejidad e incertidumbre global, mayor competitividad y productividad de la empresa en procura de establecer una visión de calidad continua.

\section{Desarrollo}

\section{El Liderazgo}

En esta perspectiva, según Montaño (2005), para abordar el tópico de liderazgo, es indispensable realizar una aproximación, primero al concepto de líder, que se abordará más adelante, y en segundo lugar como establecer los principios que rigen este proceso, el autor hace referencia a los siguientes principios:

- Principios de Legitimidad: se entiende como una situación social en donde se presenta el consentimiento tácito y explícito de los sujetos involucrados, el respeto por las normas 
formales y el reconocimiento de quienes en un momento dado, han delegado en un tercero su capacidad de intervenir directamente en la toma de decisiones y búsqueda de alternativas para el logro de los objetivos. D esta forma un buen liderazgo debe ser considerado legítimo y gozar por tanto de esa legitimidad que otros han otorgado.

- Principio de solidaridad: denota un alto nivel de integración y equilibrio interno en un grupo u organización, que implica asumir y tomar parte tanto de sus beneficios como de sus riesgos, razón por la que el liderazgo promueve las más diversas formas de solidaridad.

- Sentido de la democracia: es un principio que se basa en la libertad que los ciudadanos tienen para decidir y en la responsabilidad que poseen en la definición de los propósitos de la organización, el diseño de las estrategias para lograrlos, de acuerdo a las reglas establecidas bajo la igualdad y la equidad.

De lo anteriormente planteado, es importante resaltar que el liderazgo, guarda estrecha relación con la capacidad y habilidad de ejercer influencia sobre todas las actividades que realiza un grupo organizado y por tanto en los esfuerzos que éste realiza, tanto para definir como para alcanzar los objetivos y metas trazadas de manera colectiva.

Cabe mencionar que según Thielen (2000, citado por Chiavenato, 2006), muchos estudiosos han coincidido en que existen dos estilos de liderazgo diferentes: uno orientado hacia la realización de tareas y actividades, otros orientado al que hace o realiza las tareas o actividades y sus necesidades. El primero tiene más interés hacia la supervisión, de esta manera tiende a asegurarse de que el trabajo se realiza satisfactoriamente. El segundo, se preocupa más por establecer una relación amistosa, de confianza y respeto a quienes trata de motivar y permitirles la participación en las decisiones que le afectan.

Por tanto, los estilos de liderazgo se basan en las formas en que interactúan los dos estilos antes mencionados, de los cuales según Koontz y Weihrich (1999), se desprenden los siguientes: el líder autocrático impone y espera cumplimiento, es dogmático, se conduce por medio de la 
capacidad de premios y castigos. El líder democrático, o participativo, consulta a sus seguidores respecto de acciones y decisiones probables, alienta la participación del grupo.

El líder liberal da rienda suelta, le concede a sus seguidores independencia en sus operaciones. El líder transaccional es aquel que identifica las necesidades de sus seguidores, para cumplir sus objetivos, aclaran las funciones y tareas del grupo, toman en cuenta las necesidades sociales del grupo. Los líderes transformacionales, articulan una visión e inspiran a sus seguidores, desarrollan una capacidad para motivar a sus seguidores, al mismo tiempo que crean un ambiente favorable para el cambio.

Es de hacer notar, primero, que el uso de un estilo de liderazgo u otro dependerá de la situación y segundo, que el liderazgo que se desarrolle en las comunidades permitirá, la formación de agentes sociales capaces de ejercer el rol de líderes, cuya función más importante es que se conviertan en verdaderos motores de la transformación puesta al servicio del mejoramiento de la calidad de vida de la comunidad. Por tanto no se trata de cambiar a la gente, sino de sensibilizarla y prepararla para adaptarse a los cambios, de manera de promover e incentivar la participación para construir en equipo el logro de las metas propuestas por la organización.

Al mismo tiempo según Stelling (2005), el líder que pretende transformar una realidad que le afecta, debe estar preparado para lidiar con cambios y transformaciones en diferentes áreas y dimensiones, entre las cuales señala:

- Cambios políticos, en las fuerzas y relaciones de poder que conducen al nuevo orden social.

- Cambios institucionales, la nueva institucionalidad.

- Cambios instrumentales, en el orden organizacional, con miras a organizaciones más eficientes.

- Cambios en el tejido social, la regeneración del entramado social.

- Cambios Culturales en normas, valores y creencias.

- Cambios psicosociales, que involucran a todos los ciudadanos. 
En este sentido, se genera la posibilidad de promover acciones en favor de promover el accionar gerencial en pro de articular un estilo de organización basada en la mejora continua, así mismo, Thielen (2000, citado por Chiavenato, 2006), indica que es muy difícil precisar las características de un buen líder, puesto que en ocasiones los rasgos que definen al líder personalmente, necesitan supeditarse a las circunstancias y a las exigencias del grupo. Sin embargo la autora señala las siguientes características del buen líder, que son importantes para garantizar la efectividad del liderazgo:

- Flexibilidad: es necesario que tenga la capacidad de trabajar en equipo y adoptar posiciones que favorezcan al grupo antes que a su beneficio personal.

- Comprensión de las necesidades humanas para poder actuar con justicia y equidad.

- Alto sentido de honestidad.

- Energía intelectual y física.

- Accesible a su grupo de trabajo a fin de establecer nexos cordiales de amistad y respeto mutuo.

- Adaptación al cambio.

- Iniciativa para emprender nuevos proyectos y resolver situaciones diversas.

- Dispuesto al aprendizaje continuo.

- Visión de futuro con lo cual podrá mantenerse actualizado con las exigencias del presente, pero animando a su equipo a estar dispuesto a dar un paso hacia adelante.

Esto indica la posibilidad de promover un accionar en favor de articular el perfil de líder que la organización necesita para su continuo crecimiento, en pro de articular acciones favorables en favor de estar a la vanguardia de los retos globales, los cuales obligan a las empresas a mejorar permanentemente, actualizarse para no perecer en el mercado voraz desde el punto de vista competitivo. 


\section{Metodología de la investigación}

La investigación de tipo explicativa, con un diseño cuasi experimental, lo que permitió comparar la diferencia obtenida en el momento inicial y final, tanto en grupo experimental y control, con la finalidad de comparar los resultados de ambos momentos, generándose así el análisis estadístico para determinar la eficacia o no del tratamiento aplicado. Hernández, Fernández y Baptista (2008) explican que:

Las puntuaciones de las pre pruebas pueden usarse para fines de control en el experimento, al compararse las pre pruebas de los grupos se puede evaluar qué tan adecuada fue la aleatorización. La segunda ventaja reside en que se puede analizar el puntaje ganancia de cada grupo (la diferencia entre la pre prueba y la postprueba) (p. 193).

Siguiendo este orden de ideas, se realizó una comparación donde se estableció las diferencias entre los grupos y los resultados obtenidos. En consecuencia, el diagrama de este diseño, de acuerdo a Hernández, Fernández y Baptista (2008) se estableció de la siguiente manera:

G1 = Grupo al cual se le aplicó el tratamiento

01 = Momento de la pre prueba del G1

$\mathrm{X}=$ Tratamiento aplicado a $\mathrm{G} 1$

02 = Momento de la post prueba a G1

G2 = Grupo al cual no se le aplicó el tratamiento

03 = Momento de la pre prueba del G2

- = Tratamiento no aplicado a G2

04 = Momento de la post prueba a G2

La prueba fue aplicada a la población de la empresa suministros Klamer, ubicados en la ciudad de Portoviejo de la provincia de Manabí - Ecuador, conformada por 63 personas, las cuales se dividieron en dos grupos al azar, comtem0plados del siguiente modo: G1 conformado por 31 personas y G2 conformado por 32 personas, lo cual permitió establecer el recorrido investigativo, desarrollado del siguiente modo:

Semana 1: Presentación del tratamiento a ambos grupos, selección del grupo control y experimental. 
Semana 2: Aplicación de pre prueba a ambos grupos.

Semana 3 a 7: Aplicación de tratamiento.

Semana 8: Aplicación de pos prueba a ambos grupos.

Semana 9: Generación de resultados estadísticos para determinar la eficacia del tratamiento.

\section{Resultados}

\section{Cuadro 1}

Comparación de pre y pos prueba

\begin{tabular}{|c|c|c|c|c|c|c|}
\hline & \multicolumn{6}{|c|}{ Valor de prueba $=5$} \\
\hline & \multirow[b]{2}{*}{$t$} & \multirow[b]{2}{*}{ gl } & \multirow[b]{2}{*}{ Sig. (bilateral) } & \multirow{2}{*}{$\begin{array}{l}\text { Diferencia de } \\
\text { medias }\end{array}$} & \multicolumn{2}{|c|}{$\begin{array}{c}95 \% \text { de intervalo de confianza } \\
\text { de la diferencia }\end{array}$} \\
\hline & & & & & Inferior & Superior \\
\hline G2PRE & $-127,000$ & 31 &, 000 & $-3,96875$ & $-4,0325$ & $-3,9050$ \\
\hline G2Pos & $-127,000$ & 31 &, 000 & $-3,96875$ & $-4,0325$ & $-3,9050$ \\
\hline G1Pos & $-2,334$ & 30 & .026 & -.29032 & -.5443 & -.0363 \\
\hline
\end{tabular}

Se realizó una prueba de hipótesis utilizando un nivel de significancia de 5\%. Las hipótesis planteadas son:

Nula H0: No existe diferencias significantes entre ambos grupos, lo que es igual no funcionó el tratamiento.

Alterna H1: Existen diferencias significantes entre ambos grupos, lo que es igual, sí funcionó el tratamiento.

Se efectuó una prueba T de Student, por medio del programa SPSS 25.0, describiéndose del siguiente modo:

Por cuanto en la tabla de "pruebas independientes" (pre test), el valor de Levene es < 0,05 entonces se asumen varianzas iguales. En dichos resultados el valor de significancia bilateral fue 
de ,000 el cual es $<0,05$, lo que indica que no existen diferencias significativas entre ambos grupos (experimental y control) para el momento del pre test.

En las filas de la tabla de "pruebas independientes" concerniente al pos test, el valor de Levene también fue de $<0,05$, por lo tanto, se asumen varianzas iguales. En este caso, el nivel de significancia bilateral cambió a, 026, lo que indica diferencias significativas en ambos grupos (experimental y control).

Se resumen que el tratamiento aplicado al grupo experimental surtió efectos positivos por cuanto existió diferencia estadística entre pre y pos prueba. Por consiguiente se procede a rechazar H0 y se acepta H1, lo que indica que el tratamiento aplicado resultó favorable a la muestra objeto de estudio.

\section{Conclusiones}

En función de los resultados obtenidos, el talento humano que participó en el grupo experimental, se encuentra en capacidad cognitiva de asumir un liderazgo gerencial en pro de promover acciones transformadoras en la organización, desde una visión compleja y sistémica de la realidad, lo cual permite contar con líderes que trabajen en equipo para la resolución de conflictos, aunados que saben que la incertidumbre es parte del proceso gerencial, aunado a la visión global de abordaje del mercado para ser cada día más competitivo, para lo cual es importante generar competencias en diversas áreas que contribuyan al fortalecimiento de la personalidad como punto de partida de un buen líder.

Trabajar en una organización basada en líderes, permite contar con pensadores estratégicos que puedan mirar más allá de los muros de la organización, previniendo la articulación de acciones que permitan anticipar las acciones negativas que puedan sobrevenir, convirtiéndolas en oportunidades para crecer integralmente en favorecer no solo la productividad organizacional, sino el propio crecimiento personal como ente motivador para articular cada día mejores acciones. 


\section{Referencias}

1. Aldana, J., Salón, M., \& Guzmán, N. (2019). Liderazgo sistémico en las competencias gerenciales docentes universitarias. CIENCIAMATRIA, 5(8), 50-74. https://doi.org/10.35381/cm.v5i8.87

2. Chiavenato, I. (2006). Administración de Recursos Humanos. McGraw Hill: México.

3. Hernández, R., Fernández, C., \& Baptista, P. (2008). Metodología de la investigación. México, Mc Graw Hill Hispanoamericana. Hill Internacional.

4. Isea-Argüelles, J. J., \& Aldana-Zavala, J. J. (2017). Filosofía gerencial Kaizen dirigido al talento humano con responsabilidades gerenciales administrativas. Revista Científica FIPCAEC (Fomento De La investigación Y publicación En Ciencias Administrativas, Económicas Y Contables). ISSN: 2588-090X. Polo De Capacitación, Investigación Y Publicación (POCAIP), 2(2), 17-31. https://doi.org/10.23857/fipcaec.v2i2.15

5. Koontz, h. Weihrich, h. (1999). Administración una Perspectiva Global. (11 ${ }^{\mathrm{a}}$ Ed.). McGraw Hill. México.

6. Montaño, E. (2005): Liderazgo, Participación Comunitaria y Ciudadana. Popayán.

7. Rojas de Uzcátegui., B., Molleja, E., \& Morles, M. (2016). El autoliderazgo: Una vía para el pensamiento complejo en la gerencia pública. Revista Arbitrada Interdisciplinaria Koinonía, 1(1), 43-59. Recuperado de http://fundacionkoinonia.com.ve/ojs/index.php/revistakoinonia/article/view/14/7

8. Stelling, M. (2005): Modo Gerencial de la Política Social en la Presente Gestión Gubernamental. Escuela de Gerencia Social. Caracas- Venezuela.

\section{References}

1. Aldana, J., Salón, M., \& Guzmán, N. (2019). Liderança sistêmica nas habilidades de gestão de ensino universitário. CIÊNCIA, $5 \quad$ (8), 50-74. https://doi.org/10.35381/cm.v5i8.87 
2. Chiavenato, I. (2006). Administração de Recursos Humanos. McGraw Hill: México.

3. Hernández, R., Fernández, C., \& Baptista, P. (2008). Metodologia da Investigação. México, americano latino-americano de Mc Graw Hill. Hill International

4. Isea-Argüelles, J.J. \& Aldana-Zavala, J.J. (2017). Filosofia de gerenciamento Kaizen voltada para o talento humano com responsabilidades de gerenciamento administrativo. Revista Científica FIPCAEC (Promoção de pesquisa e publicação em Ciências Administrativas, Econômicas e Contábeis). ISSN: 2588-090X. Centro de Treinamento, Pesquisa e Publicação (POCAIP), 2 (2), 17-31. https://doi.org/10.23857/fipcaec.v2i2.15

5. Koontz, h. Weihrich, h. (1999). Administração uma perspectiva global. (11a ed.). McGraw Hill México

6. Montaño, E. (2005): Liderança, Comunidade e Participação Cidadã. Popayan.

7. Rojas de Uzcátegui., B., Molleja, E., \& Morles, M. (2016). Auto-liderança: Um caminho para o pensamento complexo na gestão pública. Revisão interdisciplinar arbitrada Koinonía, $\quad 1 \quad$ (1), 43-59. $\quad$ Recuperado de http://fundacionkoinonia.com.ve/ojs/index.php/revistakoinonia/article/view/14/7

8. Stelling, M. (2005): Modo de Gerenciamento de Política Social na Administração Atual do Governo. Escola de Gestão Social. Caracas Venezuela.

C2019 por los autores. Este artículo es de acceso abierto y distribuido según los términos y condiciones de la licencia Creative Commons Atribución-NoComercial-Compartirlgual 4.0 Internacional (CC BY-NC-SA 4.0) (https://creativecommons.org/licenses/by-nc-sa/4.0/). 